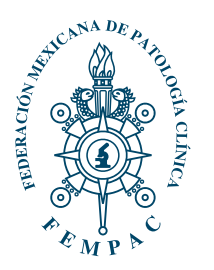

\title{
Nuevo coronavirus: la urgente necesidad de realizar una selección adecuada del ensayo clínico
}

\author{
Novel coronavirus: the urgent need to make an \\ adequate selection of laboratory assay
}

Sierra García-de Quevedo Julio,* Ramos Coronado Alfonso, ${ }^{\ddagger}$ Aguirre-Langle Eduardo§

Palabras clave: Coronavirus, SARS-CoV-2, ensayo clínico.

Keywords: Coronavirus, SARS-CoV-2, clinical trial.

\section{* Jefe del Servicio de Inmunoanálisis de la Unidad de Patología Clínica. ‡ Médico Residente de Patología Clínica de 2do año. Hospital Universitario «Dr. José Eleuterio González». Universidad Autónoma de Nuevo León. Monterrey, Nuevo León. \\ $\S$ Director General, Asesores Especializados en Laboratorios, S.A. de C.V. Puebla, Puebla.}

Correspondencia: Julio Sierra García-de Quevedo

Av. México Núm. 2341, Col. Ladrón de Guevara, 44650,

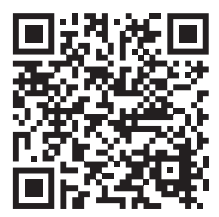

\section{RESUMEN}

La pandemia del SARS-CoV-2 ha inundado el mercado de pruebas serológicas que, por su reciente fabricación, no han sido probadas en ensayos multicéntricos, estudios comparativos, poblacionales y de validación en su precisión clínica. La selección de la prueba que mejor se adapte a las necesidades de salud poblacional y a los recursos de cada laboratorio es una necesidad prioritaria, por lo que el Comité Evaluador de Pruebas Inmunológicas (CEPI) del Consejo Mexicano de Empresas de Diagnóstico Médico (COMED) ha elaborado una guía para facilitar la selección de la prueba. Es necesario tomar en cuenta las regulaciones sanitarias nacionales e internacionales, tipo de muestra, especificaciones preanalíticas comunes a los ensayos clínicos, especificaciones de desempeño y evaluación de la precisión. Este algoritmo ha sido evaluado por diferentes integrantes de CEPI con al menos 12 insertos diferentes, concluyendo que en la práctica diaria es una herramienta útil para la mejor selección del inmunoensayo.

\section{ABSTRACT}

The SARS CoV-2 pandemic has flooded the market with serology diagnostic tests, that due to their recent fabrication, have not been tested in multicentric trials, comparative studies, population studies and clinical precision validation studies. Selecting a test that better adapts to the needs of the population health, as well as the resources of every laboratory, has become a primary necessity. Therefore, Immunological Test Evaluating Committee (CEPI) represented by the Mexican Counsel of Diagnostic Enterprises (COMED) has elaborated a guide to facilitate selecting a test by taking into consideration national and international sanitary regulations, type of sample, preanalytical specifications common to the clinical assays as well as performance and precision evaluations. This algorithm has been evaluated by multiple members of CEPI, assessing at least 12 different inserts concluding that in daily practice, it is a useful tool for the best selection of the immunoassay.
T a reciente pandemia del SARS-CoV-2 ha $\downarrow$ venido a inundar el mercado de pruebas serológicas de diferentes marcas comerciales que por su reciente fabricación aún no han sido probadas en ensayos multicéntricos, estudios comparativos, poblacionales o de validación de su precisión obtenida en el terreno clínico.

Estas pruebas han sido desarrolladas en dos vertientes: serológica y moleculares. Existen más de 250 marcas registradas y más de 600 ensayos clínicos diferentes registrados por la Fundación para la Innovación de Nuevos Diagnósticos (FIND) de Ginebra, Suiza. ${ }^{1} \mathrm{La}$ organización Global Preparedness Monitoring Board, organización paralela a la OMS, ha destinado una partida de $\$ 500$ millones de dólares para el desarrollo de pruebas diagnósticas de los 8 billones encaminados a mitigar el impacto que la pandemia del SARS-CoV- ${ }^{2}$ ha ocasionado en los sistemas de salud de los diferentes países afectados. Por lo tanto, es de

Citar como: Sierra GQJ, Ramos CA, Aguirre-Langle E. Nuevo coronavirus: la urgente necesidad de realizar una selección adecuada del ensayo clínico. Rev Mex Patol Clin Med Lab. 2020; 67 (2): 89-92. doi: 10.35366/95553 
Guadalajara, Jalisco, México.

Tel: (33) 3669-0310 E-mail: julio.sierra@ upc.com.mx

Recibido: 30/06/2020 Aceptado: 07/09/2020 suponerse que el desarrollo y la afluencia de nuevas pruebas seguirán adelante.

La presión social con base en el derecho a la salud y a la información que ejerce la sociedad, aunada a la presión económica ocasionada por el n-COVID, demandan cada vez más la implementación de pruebas «rápidas» en los laboratorios clínicos que se enfrentan con la dificultad de tomar una decisión bajo el desconocimiento del rendimiento de los nuevos ensayos autorizados o no por el estado de emergencia.

Se comprenderá la selección de la prueba que mejor se adapte a las necesidades de salud poblacional y se adecúe a los recursos de cada laboratorio se vuelve prioritaria. Para ello, realizar un análisis exhaustivo partiendo de la fase preanalítica con la información disponible es indispensable. Analizar su indicación clínica y metodología empleada, conocer su precisión y concordancia obtenidas por el fabricante y la adecuada descripción en la interpretación del resultado son la base para el éxito al momento de la implementación de la prueba.

Ante esta necesidad, el Comité Evaluador de Pruebas Inmunológicas (CEPI) del Consejo Mexicano de Empresas de Diagnóstico Médico (COMED) ha elaborado una guía de ayuda para facilitar la selección de la prueba acorde a los recursos del laboratorio. La guía toma en cuenta la aprobación del organismo regulatorio y de otros organismos, algunos aspectos de la fase preanalítica con base en la información del fabricante y sus características de desempeño y el desempeño obtenido in situ. Son cinco rubros con diferentes indicadores en forma de algoritmo. Cada rubro tiene requisitos indispensables que deben cumplirse para seguir adelante. Si éstos no se cumplen al final de la evaluación o no cumple con los estándares de calidad de cada laboratorio no vale la pena su implementación (Anexo 1). El primer paso se refiere a las regulaciones sanitarias nacionales e internacionales con que cuente la prueba. El segundo paso contempla el tipo de muestra y otras posibilidades de aplicación. En el tercer paso se establecieron 11 especificaciones preanalíticas indispensables comunes a toda prueba con un grado mínimo de calidad. ${ }^{3}$ Las especificaciones de desempeño en cuanto a su precisión y concordancia se establecen en un cuarto paso y por último valorar la precisión o exactitud, que debe ser acorde a la del fabricante.

Algunos requisitos son indispensables para poder pasar a la siguiente fase. Por ejemplo, al momento de implementación del ensayo clínico, éste debe contar con la aprobación sanitaria. Este rubro parte de un principio regulatorio. En México tiene que contar con la autorización sanitaria del país, la Comisión Federal para la Protección de Riesgos Sanitarios (COFEPRIS), si no fuera así, puede exponerse a una sanción. Puede contar con una certificación por la Food and Drug Administration (FDA) o por la Comisión Europea (CE) como producto conforme. Si es así, cumple con dos puntos, pero el primero es indispensable. Como sugerencia de este comité se sugiere seleccionar aquellas pruebas que han calificado al menos con $80 \%$ de los puntos especificados.

En el segundo rubro debe constar el o los tipos de muestra y recolección. Este aspecto, aunque obvio, es fundamental para evitar errores que influirán en el resultado; debe sumar al menos un punto. En el rubro tres, un aspecto esencial es identificar la intención de la prueba. Cuando lo que se quiere es detectar el agente causal en su diseño metodológico, se recurrirá a técnicas moleculares y así identificar componentes estructurales del agente causal. $\mathrm{Si}$ lo que se requiere es detectar la respuesta inmunológica al agente infeccioso, entonces necesita de una metodología serológica. En el caso de estas pruebas, para el SARS-CoV-2 existen varias técnicas de detección de los anticuerpos (Ac) producidos. En su mayoría la oferta actual es por inmunocromatografía de flujo lateral (FL). Éstas pueden ser de manera directa para el antígeno (Ag) o indirecta para los Ac. Las indirectas contienen en la almohadilla el suero monoclonal contra Ac IgG o IgM, utilizando como conjugado oro coloidal. Son pruebas de baja complejidad que no requieren equipo especializado, ni personal altamente capacitado para su ejecución, de las cuales, por el momento no hay reportes de estudios comparativos entre diferentes marcas comerciales.

Las pruebas de alta complejidad requieren de equipo especializado, personal entrenado y un control estricto de su desempeño. Las que se han aprobado son pruebas inmunoenzimáticas indirectas que detectan la presencia de Ac lgA, 
IgM o IgG sensibilizadas con antígenos de proteínas estructurales (S-1 y S-2) o de la nucleocápside (N). También están las inmunoenzimáticas por ELISA (Enzyme Linked Immunosorbent Assay); actualmente, sólo EIA (Enzyme Immune Assay) y por quimioluminiscencia (CMIA) con resultados cualitativos. Conviene recordar que en el caso específico del virus SARS-CoV-2 las pruebas serológicas tienen una utilidad limitada a causa de la curva de la respuesta inmune ante la infección. ${ }^{4}$ Dada la sensibilidad de las pruebas nasofaríngeas cuando no es bien realizada la técnica de hisopado, la utilización de pruebas serológicas está indicada en la detección de posibles contagios o de pacientes sintomáticos con PCR negativo. ${ }^{5}$ Por diferentes publicaciones bibliográficas se sabe que la formación de anticuerpos IgG e IgM se dan de manera casi simultánea, por lo que el desarrollo de las pruebas automatizadas se ha encaminado a este primer isotipo de inmunoglobulinas. ${ }^{6} \mathrm{Es}$ importante identificar qué proteína antigénica se ha utilizado en la fabricación del reactivo, ya que de ello dependerá la aplicación clínica de la prueba. Por ejemplo, se pueden detectar anticuerpos contra las proteínas estructurales del virus, como en el caso de las proteínas SPIKE S-1 y S-2, que inducirá la formación de anticuerpos neutralizantes necesarios, o contra las proteínas $\mathrm{N}$ de la nucleocápside que no formarán este tipo de $\mathrm{Ac}^{7}$ y la aplicación clínica será diferente.

En el desempeño de calidad de la prueba, en cuanto a la sensibilidad y especificidad, el resultado puede inducir a falsos negativos si el periodo de exposición al agente causal es muy corto o a un falso positivo en el caso de exposición a otros coronavirus humanos y presentar reacciones cruzadas. ${ }^{8}$

Este grupo recomienda que, de los 23 parámetros a evaluar, se cumplan con los requisitos indispensables y con al menos $80 \%$ de los cuatro primeros parámetros para evaluar posteriormente el índice de desempeño intralaboratorial. Este algoritmo ha sido evaluado por diferentes integrantes de CEPI con al menos 12 insertos diferentes, con lo que concluyen que en la práctica diaria es una herramienta útil ante la avalancha de pruebas que existen y que seguirán fluyendo en el país. ${ }^{9}$

\section{REFERENCIAS}

1. Foundation for Innovative New Diagnostics (FIND). From our CEO COVID-19: diagnostics in the global spotlight (about 1 screen) [Internet]. Geneva [2020 Apr 17]. Available in: www.finddx.org/ newaroom/ceo-17apr20

2. Global Preparedness Monitoring Board (GPMB). A world at risk: annual report on global preparedness for health emergencies [Internet]. Geneva: World Health Organization; 2019. Available in: www.apps.who.int/gpmb/assets/pdf/covid_19_press-Realease_ cmp-9mar.pdf

3. Leeflang MMG, Allerberger F. How to: evaluate a diagnostic test. Clin Microbiol Infect. 2019; 25 (1): 54-59. doi: 10.1016/j. cmi.2018.06.011.

4. Cheng MP, Papenburg J, Desjardins M et al. Diagnostic testing for severe acute respiratory syndrome-related coronavirus 2: a narrative review. Ann Intern Med. 2020; 172 (11): 726-734. doi: 10.7326/ M20-1301.

5. Guo L, Ren L, Yang S et al. Profiling early humoral response to diagnose novel coronavirus disease (COVID-19). Clin Infect Dis. 2020; 71 (15): 778-785. doi: 10.1093/cid/ciaa310.

6. Sethuraman N, Jeremiah SS, Ryo A. Interpreting diagnostic tests for SARS-CoV-2. JAMA. 2020. doi: 10.1001/jama.2020.8259.

7. Petherick A. Developing antibody tests for SARS-CoV-2. Lancet. 2020; 395 (10230): 1101-1102.

8. Okba N, Müler M, Li W, Wnag CH, Geurtsvan Kessel C, Corman $\checkmark$ et al. SARS-CoV-2 specific antibody responses in COVID-19 patients. medRxiv. 2020. doi: 10.1101/2020.03.18. 20038059.

9. https://drive.google.com/drive/folders/1CyVLBQh6BfkTgAn717Fy5SCNIhRHE7L 
Anexo 1: Algoritmo para la validación de pruebas de laboratorio.

\begin{tabular}{|c|c|c|c|c|}
\hline Nombre de la prueba: & Realizó: & & & \\
\hline Fecha: & Autorizó: & & & \\
\hline Regulaciones sanitarias & & & & \\
\hline & Sí & No & & mentario \\
\hline 1. Aprobación regulatoria por las autoridades nacionales & 1 & 0 & Satisfactorio & No satisfactorio \\
\hline 2. Aprobaciones regulatorias internacionales & 1 & 0 & $\geq 1$ & no se especifica el tipo \\
\hline & Total 2 & 0 & 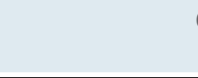 & $\begin{array}{l}\text { de muestra, no puede } \\
\text { continuar }\end{array}$ \\
\hline Tipo de muestra & & & & \\
\hline Requisito indispensable dependiendo de la aplicación & Especifique & & & \\
\hline de la prueba: & Sí & No & & omentario \\
\hline 1. Suero & 1 & 0 & Satisfactorio & No satisfactorio \\
\hline 2. Orina & 1 & 0 & & Si no se especifica el \\
\hline 3. LCR & 1 & 0 & $\geq 1$ & tipo de muestra, no \\
\hline 4. Heces & 1 & 0 & & puede continuar \\
\hline 5. Otro & 1 & 0 & & \\
\hline & Total 5 & 0 & & \\
\hline Especificaciones de uso & Sí & No & & mentario \\
\hline 1. Especifica la intención de la prueba & 1 & 0 & Satisfactorio & No satisfactorio \\
\hline 2. Especifica la metodología usada & 1 & 0 & 10 & $<9$ \\
\hline 3. Número de lote & 1 & 0 & & \\
\hline 4. Temperatura almacenamiento & 1 & 0 & & \\
\hline 5. Fecha de caducidad & 1 & 0 & & \\
\hline 6. Instrucciones de uso & 1 & 0 & & \\
\hline 7. Pasos y procedimientos requeridos & 1 & 0 & & \\
\hline 8. Interferencias & 1 & 0 & & \\
\hline 9. Reacciones cruzadas & 1 & 0 & & \\
\hline 10. Interpretación de resultado & 1 & 0 & & \\
\hline & Total 10 & 0 & & \\
\hline & $\mathrm{n} \geq 30$ & & & \\
\hline Especificaciones óptimas de desempeño & Sí & No & & mentario \\
\hline 1. Sensibilidad y especificidad & 1 & 0 & Satisfactorio & No satisfactorio \\
\hline 2. Precisión diagnóstica & & 0 & $\geq 2$ & Si no se especifican los \\
\hline 3. Bibliografía & 1 & 0 & & puntos siguientes, no \\
\hline 4. Revisión de uso en la literatura & & 0 & & puede seguir adelante \\
\hline & Total 2 & 0 & & el algoritmo \\
\hline Especificaciones de desempeño en la rutina & Sí & No & & mentario \\
\hline 1. Sensibilidad y especificidad obtenida & 1 & 0 & Satisfactoric & No satisfactorio \\
\hline 2. Precisión diagnóstica obtenida & & 0 & $\geq 1$ & Si en el punto 1 no \\
\hline & Total 2 & 0 & & se obtiene $\geq \%$ no \\
\hline & & & & se puede seguir \\
\hline & & & Indice & de desempeño \\
\hline & & & 20 & de 21 \\
\hline $\begin{array}{l}\text { Revisado y aprobado por: COMED/CEPI } \\
\text { Adaptado por: Dr. José Julio Sierra García de Quevedo. Kosack CS, } \\
\text { Page AL, Klatser PR. A guide to aid the selection of diagnostic tests. } \\
\text { Bull World Health Organ. 2017; } 95 \text { (9): 639-645. }\end{array}$ & & & $\begin{array}{l}\text { Si no cumple co } \\
\text { requisitos crític } \\
\text { no se recomieno } \\
\text { evaluación }\end{array}$ & $\begin{array}{l}\text { los } \\
\text { os, } \\
\text { la su }\end{array}$ \\
\hline
\end{tabular}

